\title{
Tangence
}

\section{Des mouvements de la nature à la mise en scène du corps libertin : la savante éloquence d'une Fille de joye}

\section{Marc André Bernier}

Numéro 60, mai 1999

L'éloquence du corps sous l'Ancien Régime

URI : https://id.erudit.org/iderudit/008082ar

DOI : https://doi.org/10.7202/008082ar

Aller au sommaire du numéro

Éditeur(s)

Presses de l'Université du Québec

ISSN

0226-9554 (imprimé)

1710-0305 (numérique)

Découvrir la revue

Citer cet article

Bernier, M. A. (1999). Des mouvements de la nature à la mise en scène du corps libertin : la savante éloquence d'une Fille de joye. Tangence, (60), 84-94.

https://doi.org/10.7202/008082ar d'utilisation que vous pouvez consulter en ligne. 


\title{
Des mouvements de la nature à la mise en scène du corps libertin : la savante éloquence d'une Fille de joye
}

\author{
Marc André Bernier, Université du Québec à Trois-Rivières
}

Dans un passage resté célèbre de son troisième livre du traité De l'orateur, Cicéron soutient que la nature a voulu que les choses les plus utiles soient en même temps celles qui ont le plus de grâce. Regardez les arbres, poursuit le texte: tronc, branches, scions et feuilles, tout y est utile, rien n'y est sans grâce ${ }^{1}$. À plusieurs siècles d'intervalle, Fougeret de Monbron traduit et adapte Fanny Hill (1748-1749), ouvrage libertin que l'on doit au romancier anglais John Cleland. Devenu en français La fille de joye ${ }^{2}$, ce roman, écrit son traducteur en 1751, est "quintessencié de l'anglois" et, dans cette expression heureuse, se laisse pressentir l'éloquence d'une écriture qui va s'inventer à partir de réminiscences érudites. En effet, ces branches et ces scions que Cicéron évoquait pour illustrer l'idée qu'il se faisait de la nature vont donner lieu à la reprise libertine de ce motif, alors que Fanny, la narratrice encore jeune et innocente de ce roman, raconte dans

1 Cicéron, De l'orateur, Paris, Société d'Édition Les Belles Lettres, "Collection des Universités de France", 1971, III, XLV/XLVI-178/179, p. 72-73 [éd. crit. Henri Bornecque et trad. Edmond Courbaud et Henri Bornecque]. Le passage se lit comme suit: "Sed ut in plerisque rebus incredibiliter hoc natura est ipsa fabricata, sic in oratione, ut ea, quae maximam utilitatem in se continerent, plurimum eadem haberent uel dignitatis uel saepe etiam uenustatis [...]. Quid in eis arboribus? in quibus non truncus, non rami, non folia sunt denique nisi ad suam retinendam conseruandamque naturam; nusquam tamen est ulla pars nisi uenusta" "Mais il en est du discours comme de presque toutes les choses; la nature, d'elle-même, par un phénomène merveilleux, a voulu que les choses les plus utiles offrissent en même temps le plus de majesté ou souvent même de grâce [...]. Et les arbres? Tronc, branches, feuilles ne servent en définitive qu'à y conserver et entretenir la vie; cependant il ne se trouve aucun élément qui n'ait sa grâce").

2 Fougeret de Monbron, La fille de joye. Ouvrage quintessencié de l'anglois, Lampsaque, 1751; John Cleland, Fanny Hill, la fille de joie. Récit quintessencié de l'anglais par Fougeret de Monbron, Arles, Actes Sud, coll. "Babel", 1993; c'est à la réédition moderne que je me référerai dans les pages qui suivent. 
quelles circonstances l'une de ses compagnes mieux avisée en vint à porter la main sur cet "agréable réduit des plus délicieuses sensations " :

Ses doigts, précise Fanny, jouaient et tâchaient d'allonger les tendres scions de cette charmante mousse que la Nature a fait croître autant pour l'ornement que pour l'utilité. ${ }^{3}$

À la faveur de cette description, la représentation du corps sollicite à la fois une docte éloquence et une conception savante de la nature. Coquette, la nature se plaît à prodiguer les grâces et les ornements: sous cette figure, elle offre les mêmes traits que lui prêtait jadis Cicéron, si bien que tout le passage illustre à nouveau une conception de la nature que je qualifierais volontiers d' "oratoire" et qui est encore largement accréditée au $\mathrm{XVIII}^{\mathrm{e}}$ siècle ${ }^{4}$. Dans la Fille de joye, les références à la nature ne se réduisent pourtant pas à soutenir une représentation du corps qui ne prêterait à celui-ci que la beauté un peu frivole de ces "charmantes mousses" ou de ces "tendres scions". Que l'on songe, par exemple, à la première réflexion de Fanny sur laquelle s'ouvre le roman: "Tu veux, écrit-elle à sa correspondante, [...] que je retrace à tes yeux les égarements de ma première jeunesse [...]. La vérité guidera ma plume [...]: je peindrai les choses d'après nature, sans craindre de violer les lois de la décence " ${ }^{5}$. La suite du texte ne dément pas cette ambition initiale et se résume en conséquence à une suite d'aventures licencieuses attentives à "peindre", suivant l'expression de Fanny, le corps de chaque personnage se livrant à ses désirs. Seulement, la description du corps et la représentation de ses mouvements "d'après nature"

3 Fougeret de Monbron, op. cit., p. 18.

4 "C'est une femme", déclare, par exemple, Diderot à propos de la nature, et cette femme "aime à se travestir" (L'interprétation de la nature, Paris, Hermann, 1981, tome IX, p. 38 [éd. crit. Jean Varloot]). De même, on lit, dans Les lauriers ecclésiastiques, ou Campagnes de l'abbé de $T^{* * * *}$ du chevalier de La Morlière, que le cours capricieux conduisant à quelque "bonne fortune" tient précisément à "ce fond de frivolité qui est dans la nature" (Luxuropolis, De l'Imprimerie ordinaire du clergé, 1748, p. 21). On pourrait multiplier les exemples: à chaque fois, peut-être aurait-il lieu de supposer que cette "conception oratoire" de la nature participe d'une épistémè qui, encore au XVIII ${ }^{\mathrm{e}}$ siècle, reste dominée par ce que Claire Salomon-Bayet appelait une "science baroque"; voir ici L'institution de la science et l'expérience du vivant. Méthode et expérience à l'Académie royale des sciences, 16661793, Paris, Flammarion, coll. "Science", 1978, p. 136 et sqq.

5 Fougeret de Monbron, op. cit., p. 9. 
86

supposent le concours d'une prose éloquente, laquelle s'invente à son tour à partir d'un savoir portant sur l'idée même de "nature". Ce savoir, on le verra, se rattache, d'une part, à un naturalisme vitaliste qui, hérité des Anciens et des "libertins érudits" du $\mathrm{XVII}^{\mathrm{e}}$ siècle, suppose à la nature force et fécondité. D'autre part, on examinera en quoi la mise en scène du corps libertin ne consiste pas simplement à piller d'anciens motifs naturalistes, mais tire également parti des conceptions mécanistes dont se réclament les sciences physiques et naturelles au moment où paraît La fille de joye.

Les corps libertins que Fanny s'est proposé de peindre se présentent d'abord sous une figure où perce sans cesse un naturalisme dont les traits essentiels sont tirés d'une certaine idée de la nature qu'avaient cultivée les Anciens. L'opinion suivant laquelle la nature se définit en tant que principe actif et puissance primordiale repose, on le sait, sur les conceptions les plus anciennes: celles, bien sûr, des philosophes présocratiques de l'école ionienne dont le principal objet fut, précisément, la question de la jusis: mais aussi sur celles des épicuriens et, en particulier, de Lucrèce ${ }^{6}$. Reprises aux Anciens par les philosophes de la Renaissance, de telles conceptions accréditèrent l'idée d'un principe vital inhérent à une natura naturans, c'est-à-dire à une "nature naturante" variant et renouvelant sans cesse ses productions. Sous cette forme, le naturalisme devait encore trouver, au siècle des Lumières, "des prolongements vivaces", lesquels perpétuent "au temps de Fontenelle et des Bernouilli la mentalité de Cardan ou de Bruno"7, comme l'a déjà souligné à juste titre Jean Ehrard. Pour s'aviser de cette persistance, on n'aurait qu'à songer de surcroît à la brillante fortune du De natura rerum de

6 Sur cette question, voir, entre autres, la préface de J.-P. Dumont à son édition des Présocratiques (Paris, Gallimard, 1988, p. XIII). Sur la fortune de cette conception dans la philosophie latine, voir également l'introduction de José Kany-Turpin à son édition de Lucrèce, De la nature. De rerum natura (Paris, Aubier, "coll. Bibliothèque philosophique bilingue", 1993, p. 25 et sqq.)

7 Jean Ehrard, L'idée de nature en France dans la première moitié du XVIII ${ }^{e}$ siècle, Paris, Albin Michel, "Bibliothèque de l'Évolution de l'Humanité", 1994 [1963], p. 50; voir, en particulier, les sections intitulées "Sainte et Admirable Nature...!" et "Idolum Naturce...!", première partie, $\mathbb{\$}$ et $\$ 4$, p. 45-59. Voir aussi Michel Foucault, "La prose du monde", dans Les mots et les choses, Paris, Gallimard, coll. "Bibliothèque des sciences humaines ", 1986 [1966], p. 32-59. 
Lucrèce dans la libre pensée de l'âge classique, depuis les libertins érudits du $\mathrm{XVII}^{\mathrm{e}}$ siècle, qui s'inscrivent dans la mouvance de Gassendi, jusqu'à l'ouvrage que Diderot fait paraître en 1753 avec pour titre De l'interprétation de la nature ${ }^{8}$.

Outre le célèbre prologue du traité de Lucrèce où celui-ci évoque d'emblée cette "volupté " que prodigue la "Vénus Nourricière" 9 , j'aimerais rappeler un exemple précis, à même de rendre sensibles ces remarques liminaires. Au Livre IV se retrouve ainsi la description de l'un de ces spectacles où se déploie la force primitive de la nature, alors que Lucrèce s'emploie à décrire l'éveil des désirs que suscite Vénus:

Au détroit fougueux de la vie, dès que s'épanche en nous

la semence première, le jour de sa maturation, de l'extérieur confluent les images de divers corps, promesses d'un beau visage et d'un teint éclatant, qui excitent les régions gonflées par la semence [...]

Ainsi de l'homme atteint par les traits de Vénus que lui lance le garçon aux membres féminins ou la femme dont tout le corps darde l'amour: il tend vers qui le frappe et brûle de l'étreindre, de jeter la liqueur de son corps dans le sien, car son désir muet lui prédit le plaisir. [...]

Tels les amants, jouets des images de Vénus [...]

Cupides, leurs corps se fichent, ils joignent leurs salives, [...]

Enfin jaillit le désir concentré en leurs nerfs, leur violente ardeur s'apaise un court instant, puis un nouvel accès de rage et de fureur les prend [...] Jamais les oiseaux, les fauves, les bestiaux petits ou gros, les juments ne pourraient se soumettre aux mâles si leur nature, brûlant, débordant, n'entrait en rut et ne jouissait du plaisir donné aux assaillants. ${ }^{10}$

8 Sur les libertins érudits du $\mathrm{XVII}^{\mathrm{e}}$ siècle et sur l'influence que devait exercer Lucrèce sur la pensée de Gassendi ou de Gabriel Naudé, de La Mothe le Vayer ou encore de Molière, voir l'ouvrage célèbre de René Pintard, Le libertinage érudit dans la première moitié du XvII siècle, Paris, Boivin et Cie, 1943.

9 Ici, il s'agit évidemment des deux premiers vers de l'Hymne à Vénus sur lequel s'ouvre tout l'ouvrage: "Aeneadum genetrix, bominum diuomque uoluptas, / alma Venus" ("Mère des Énéades, volupté des hommes et des dieux / Alme Vénus"); Lucrèce, op. cit., I, v. 1-2, p. 52.

10 Lucrèce, op. cit., IV, 1030-1208, p. 299 et sqq. Le texte latin se lit comme suit: "Tum quibus aetatis freta primitus insinuatur / semen, ubi ipsa dies membris matura creauit, / conueniunt simulacra foris e corpore quoque / 
$\mathrm{Au}$ "détroit fougueux de la vie", la nature se déclare, le désir jaillit et voici maintenant des corps gonflés de semence, "jouets des images de Vénus " désormais gagnés par la violence, la rage et la fureur de leurs transports, tels ces fauves et ces bestiaux qu'entraîne une force aveugle - celle d'une nature ardente "entrant en rut". Du reste, tout un bestiaire concourt ici à illustrer le rendu de cette pulsation vitale de la matière, emportée dans sa course amoureuse et emportant les corps dans une violence sans frein, brûlante et débordante. En regard de ce tableau naturaliste, examinons maintenant la manière dont est représenté le corps dans un épisode que nous rapporte Fanny dans la Fille de joye. Celle-ci évoque alors un "trait de débauche" où devait se distinguer Émilie, l'une de ses compagnes à qui était venue la fantaisie de vérifier par l'expérience l'axiome suivant lequel la "nature" compenserait toujours, chez un individu, l'imbécilité de l'esprit par quelque vertu plus secrète. Dick, un jeune gueux, "insensé et bègue", nous dit le texte, vient à s'offrir et, dès lors,

J'eus même la vanité de vouloir être la première à faire la vérification des pièces. [...] et m'étais par gradation saisie du véritable et sensible végétatif, qui loin de se retirer au toucher de mes doigts s'allongeait et se gonflait pour les rencontrer. [...] J'avoue qu'il n'était guère possible de rien voir de plus superbe. Aussi ma lascive compagne, ravie en admiration, [...] me l'ôta brusquement [...] Le fier agent animé par le puissant aiguillon du plaisir devint si furieux, qu'il me fit trembler pour la patiente. Son visage était tout en feu: ses yeux étincelaient: il grinçait des dents. Tout son corps agité d'une impétueuse rage faisait voir avec quel excès de force la nature opérait en lui. Tel on voit un jeune taureau sauvage que l'on a poussé à bout, fouler aux pieds, frapper des cornes tout ce qu'il rencontre : tel, le forcené Dick brise, rompt tout ce qui s'oppose à son passage. [...] Dick machinalement gouverné par la partie animale la

nuntia praeclari uoltus pulchrique coloris, / qui ciet inritans loca turgida semine multo [...] Sic igitur Veneris qui telis accipit ictus, / siue puer membris muliebribus hunc iaculatur, / seu mulier toto iactans e corpore amorem, / unde feritur eo tendit, gestitque coire, / et iacere umorem in corpus de corpore ductum; / namque uoluptatem praesagit muta cupido. [...] sic in amore Venus simulacris ludit amantis [...] adfigunt auide corpus, iunguntque saliuas / [...] Tandem ubi se erupit neruis conlecta cupido, / parua fit ardoris uiolenti pausa parumper; / inde redit rabies eadem et furor ille reuisit, / [...] Nec ratione alia uolucres, armenta, feraeque, / et pecudes, et equae maribus subsidere possent, / si non, ipsa quod illarum subat ardet abundans / natura, et Venerem salientum laeta retractat. ". 
pince, la mord et la secoue avec une ardeur moitié féroce, moitié tendre. [...] Agités l'un et l'autre d'une fureur égale [...] ils auraient succombé à tant d'efforts, si la crise délicieuse de la suprême joie ne les eût arrêtés subitement et n'eût terminé le combat. ${ }^{11}$

À l'aspect des fureurs de ce "fier agent", de ces dents qui grincent, de ce corps "agité d'une impétueuse rage", on se souviendra sans doute des bestiaux de Lucrèce. À chaque fois, toute l'invention se soutient d'une amplification oratoire tirée d'un même répertoire de métaphores naturalistes, si bien qu'à la puissance élémentaire et aveugle agitant les fauves en rut de Lucrèce répond ici la force vitale avec laquelle la nature opère en Dick, lequel paraît dès lors sous la figure d'un "jeune taureau sauvage". Du moment où il s'agit de décrire le corps ou de représenter ses mouvements, tout le texte ne manque d'ailleurs jamais de recourir à ces métaphores naturalistes, si bien qu'à cet égard, il rappelle parfois davantage l'exubérance érotique de la prose renaissante des Ragionamenti de l'Arétin que les tours ingénieux de la sophistique amoureuse d'un Crébillon fils ${ }^{12}$. Telle est, par exemple, le portrait que fait Fanny du premier client qu'une maquerelle, Madame Brown, cherche à lui faire agréer:

Que l'on se réprésente, écrit Fanny, un homme de soixante ans passés [...] avec de gros yeux de bœuf, une bouche fendue jusqu'aux oreilles garnie de deux ou trois défenses au lieu de dents [...] enfin un monstre dont le seul aspect faisait horreur. ${ }^{13}$

Puis "quand on crut le bouc suffisamment prévenu de mes charmes", voici que le portrait de ce "hideux animal" ou de ce "vieux singe", suivant l'expression de Fanny, se met à s'animer:

11 Fougeret de Monbron, op. cit., p. 77-79.

12 Voir, ici, Pietro Aretino, dit l'Arétin, Les Ragionamenti, Paris, Cercle du Livre Précieux, 1959 [1536 et 1556], préface de Guillaume Apollinaire. Voici un exemple représentatif de cette prose tiré du premier dialogue entre Antonia et Nanna, alors que celle-ci raconte quelques épisodes relatifs à sa vie de nonne: "Au plus beau moment de l'histoire, le Confesseur avait retiré le bouchon de la bouteille et il voulait à toute force le mettre dans le pot à civette : la pauvrette, toute en rut, toute en luxure, [...] ne put jamais obtenir que le Néron, le Caïn, le Judas, lui plantât son poireau dans le jardinet: [...] tout gonflé de venin [...] soufflant comme un aspic sans oreilles, l'écume à la bouche, comme l'orque, il lui enfonça le plantoir dans le trou restauratif "; "Première journée dans laquelle Nanna, à Rome, sous un figuier, raconte à l'Antonia la Vie des Nonnes", p. 38.

13 Fougeret de Monbron op. cit., p. 21. 
Mes pleurs ne servirent qu'à l'enflammer davantage [...] et après m'avoir jeté la chemise par-dessus la tête, le vilain fit, soufflant et mugissant comme un taureau, des efforts qui se terminèrent par une libation involontaire dont je sentis les effets sur mes cuisses. 14

On pourrait multiplier sans peine les exemples de ces métaphores naturalistes, partout disséminées dans le texte et toujours liées à la représentation du corps. Ce sont tantôt les seins d'une certaine Polly, avec leurs "deux charmants boutons de corail" ${ }^{15}$ : ou bien l'amant de celle-ci, dont le "joyeux instrument sortait avec pompe d'un taillis épais et frisé"16: ou, enfin, quelque "monstrueuse cheville [...] dont la chaleur" ressuscite les "esprit animaux" ${ }^{17}$. En bref, il n'y a pas jusqu'aux sensations les plus intimes du corps se livrant aux plaisirs de l'amour qui ne soient évoquées grâce à quelque figure propre à rendre sensible l'idée d'une nature en rut: "Je sentis derechef une émotion si vive, écrit Fanny, qu'il n'y avait que la pluie salutaire dont la nature bienfaisante arrose ces parties-là qui pût me sauver de l'embrasement "18. Tel est enfin le contexte en regard duquel doivent se lire les descriptions qui recourent à la fable antique en associant motifs mythologiques et représentation du corps au profit d'une mise en scène susceptible de favoriser une sorte d'amplification glorieuse et païenne des désirs. Les exemples de cette sorte sont, au reste, innombrables, depuis le portrait que fait Fanny de son premier amant, où elle rappelle "toutes les grâces du fils de Vénus et l'état ravissant où la tendre Psyché le surprit lorsqu'elle le trouva endormi" ${ }^{19}$ : jusqu'à cette "pièce d'une si énorme taille, qu'on l'aurait prise pour celle du géant Polyphème " 20.

Toutefois, la représentation du corps libertin ne se réduit pas, dans ce roman, à simplement revisiter certains thèmes naturalistes légués par les Anciens, repris à la Renaissance et au XVII siècle par les libertins érudits, puis figurés dans la Fille de joye à la faveur de quelque métaphore animale ou encore par la reprise d'un motif mythologique. À chaque fois, les "peintures d'après nature" de Fanny intègrent la leçon "mécaniste" que comportent

14 Ibid., p. 23-24.

15 Ibid., p. 30.

16 Ibid., p. 31.

17 Ibid., p. 57.

19 Ibid., p. 35.

20 Ibid., p. 62. 
la physique nouvelle et les sciences naturelles au moment où paraît le roman. On ne doit pas s'en étonner: pareille entreprise participe d'un travail de relecture et de réinterprétation des Anciens dont la pensée des Lumières ne manque pas d'offrir plusieurs précédents - à propos de l'œuvre de Lucrèce, par exemple. C'est ainsi que, dans l'Histoire critique de la philosophie, brillant ouvrage d'érudition que fait paraître André François Boureau-Deslandes en 1737, on considère curieusement que Lucrèce constitue le meilleur exemple d'une "Philosophie [qui] est toute méchanique " 21 . Or cette philosophie "toute mécanique" est précisément celle dont se réclament les Lumières à la suite de Descartes et, surtout, de Newton. Suivant cette perspective nouvelle et moderne, la nature ne se confond plus avec un principe naturant - autrement dit, elle n'a plus rien d'un être comportant une fécondité créatrice. Désormais, toute son action se règle sur les lois du mouvement, la mécanique des corps célestes représentant, comme chacun sait, le paradigme auquel l'âge classique se réfère le plus volontiers. Qu'on en juge ici par cet extrait de l'article que l'Encyclopédie a consacré à la question de la nature:

Quand on parle de l'action de la nature, nous dit-on, on n'entend plus autre chose que l'action des corps les uns sur les autres, conforme aux lois du mouvement [...]. C'est en cela que consiste tout le sens du mot, qui n'est qu'une façon abrégée d'exprimer l'action des corps, \& qu'on exprimeroit peut-être mieux par le mot de méchanisme des corps. ${ }^{22}$

"L'action des corps les uns sur les autres": d'une seconde manière, les descriptions du corps dans la Fille de joye vont s'employer à figurer les lois de ce "méchanisme des corps" agisssant

21 André François Boureau-Deslandes, "Des philosophes qui ont fleuri à Rome", dans l'Histoire critique de la philosophie Ou l'on traite de son origine, de ses progrès, et des diverses Revolutions qui lui sont arrivées jusqu'à notre tems, Amsterdam, F. Changuion, 1737, tome III, Livre VII, p. 30; voici le passage où se retrouve cette constatation: "J'observerai seulement, écrit Boureau-Deslandes à propos de Lucrèce, que sa Philosophie est toute méchanique, \& par conséquent d'une beauté de recherche qui revient souvent. Rien n'existe, remarque-t'il, que le vuide \& les atomes. [...] Au moyen de leurs mouvemens, de leurs masses, de leurs figures, s'exécute l'ouvrage immense \& laborieux de la Nature".

22 "Article Nature", dans Encyclopédie, ou dictionnaire raisonné des sciences, des arts et des métiers, tome XI, p. 41 [reprint: Stuttgart-Bad Cannstatt, F. Frommann Verlag, 1966-1967]. 
92

les uns sur les autres: mais consultons un exemple où s'illustre cette nouvelle conception toute mécanique de la nature sur laquelle va se régler, comme on le verra, la représentation des mouvements physiologiques et psychologiques des personnages. La description que fait Fanny d'une scène d'amour observée à la dérobée entre sa maquerelle et un jeune amant que cette dernière destine à ses plaisirs recourt d'abord à des métaphores naturalistes, alors que le jeune homme pose "sans cérémonie ses larges mains sur les effroyables mamelles ou plutôt sur les pesantes calebasses de la mère Brown "23 : mais bientôt, ce sera la mécanique des corps en mouvement qui viendra soutenir la description, comme en témoigne la suite du texte:

le héros, poursuit Fanny, produisit au grand jour cette merveilleuse et superbe pièce qui m'avait été inconnue jusqu'alors et [...] pendant la chaleur de l'action, [....] j'eus tout le loisir de remarquer le mécanisme admirable de cette machine essentielle de l'homme. ${ }^{24}$

Au reste, pendant la chaleur d'une "action" où s'éprouvent ces corps, le seul spectacle de "cette machine essentielle" fait immédiatement ou, pour mieux dire, mécaniquement ressentir à Fanny "des chatouillements presque aussi délectables que si j'en eusse réellement joui" 25 . À vrai dire, pareilles descriptions des mouvements intimes du corps engagent une attitude intellectuelle dont le texte va sans cesse mettre en évidence les enjeux épistémologiques essentiels. Reprenons, par exemple, le passage qui relate la curieuse expérience que Fanny et sa compagne Émilie tentèrent sur le jeune Dick. La nature, en effet, n'opérait pas en lui de manière à confondre ses mouvements avec la seule fureur d'un "jeune taureau sauvage". En cette circonstance, Dick était aussi "machinalement gouverné par la partie animale" et le rendu mécaniste de ses mouvements était d'autant plus marqué que la partie animale en cause devenait un "sensible végétatif ": "Je m'étais saisie du véritable et sensible végétatif, qui loin de se retirer au toucher de mes doigts s'allongeait et se gonflait pour les rencontrer". Tirée, selon toute vraisemblance, de l'ouvrage de La Mettrie intitulé l'Homme-Plante (1748), cette métaphore se justifie d'autant mieux que la description de Fanny en fait usage de

23 Fougeret de Monbron, op. cit., p. 26.

24 Ibid., p. 27-28.

25 Ibid., p. 27. 
manière à pouvoir représenter le mouvement mécanique avec lequel ce "sensible végétatif" vient répondre à la sollicitation des doigts qui le pressent de se gonfler. Le texte anglais dont Fougeret de Monbron offre une version "quintessenciée" est encore plus éloquent sur ce point, puisqu'il parle tour à tour de "sensitive plant" 26 et, à propos de Dick, de "man-machine strongly work'd upon by the sensuel passion", ou encore de "brute-machine driven like a whirlwind" "27. Dans ce contexte, s'étonnera-t-on enfin que la critique anglo-saxonne ait déjà pu envisager l'hypothèse suivant laquelle il existerait une complicité essentielle entre "the view of sexuality presented fictionally in Fanny Hill and the view of buman nature presented in more discursive philosophical form by La Mettrie in L'homme machine "28?

Que conclure de ce parcours, si ce n'est d'abord que la représentation du corps et de ses mouvements intimes, voire l'érotisme libertin en général, ne saurait se réduire à une sorte de bagatelle pornographique. Tout semble, au contraire, indiquer à quel point libertinage d'esprit et libertinage de mours se répondent à l'occasion de ces diverses "peintures" du corps qui doivent leur couleur particulière aux savoirs qu'ils mettent en œuvre. Il y a, rappelait Michel Foucault dans Les mots et les choses, "un ordre strict de la vie libertine: toute représentation doit s'animer aussitôt dans le corps vivant du désir, tout désir doit s'énoncer dans la pure lumière d'un discours représentatif " 29 Tissée de longue main par l'histoire de la libre pensée, inscrite de surcroît dans l'ambiguité sémantique du terme "libertin" ${ }^{30}$, une

26 Voir John Cleland, Memoirs of a Woman of Pleasure. Fanny Hill, Ware, Wordsworth Editions Limited, "Wordsworth Classics", 1993, p. 192. On remarquera enfin que, dans un article qui s'attache essentiellement à analyser la scène entre Fanny, Émilie et Dick, Leo Braudy semble également voir dans cette "sensitive plant", une allusion à La Mettrie ; Fanny Hill and Materialism", Eighteenth-Century Studies, vol. IV, no 1, 1970, p. 33.

27 John Cleland, op. cit., p. 194 et p. 195: "d'homme-machine que le trouble des sens ébranle avec force"; et d'"animal-machine emporté tel un tourbillon" (je traduis).

28 Leo Braudy, "Fanny Hill and Materialism.", loc. cit., p. 21: "la conception de la sexualité s'offrant dans Fanny Hill sous les traits de la fiction et la conception de la nature humaine telle qu'elle s'offre sous une forme plus discursive et philosophique dans l'Homme machine de La Mettrie" (je traduis).

29 Michel Foucault, op. cit., p. 222.

30 Sur cette question, voir, en particulier, Gerhard Schneider, Der Libertin. Zur Geites- und Sozialgeschichte des Bürgertums im 16. und 17. Jahrhundert, Stuttgart, J. B. Metzler, 1970. 
94

pareille alliance entre licences du corps et licences de l'esprit trouvent, dans le rendu des mouvements du désir, l'occasion de multiplier des tableaux dont l'éloquence parvient à réaliser ce mélange, devenu si singulier de nos jours, entre érotisme et savoirs philosophiques. À chaque fois, les peintures licencieuses de Fanny ne peuvent, en effet, se concevoir sans la prise en compte d'une dimension cognitive, qui reste indissociable, on l'a vu, d'une sorte d'amalgame entre une conception naturaliste et une conception mécaniste de la nature. C'est ainsi qu'à des métaphores issues d'un naturalisme à l'antique, le texte applique, si j'ose dire, en surimpression, tout un ensemble de figures indissociables d'une conception mécaniste et, par conséquent, moderne de la nature. Cette forme originale de "naturalisme mécaniste" que met en scène la Fille de joye présente, au reste, le mérite étonnant d'offrir une conception unifiée des deux grandes traditions libertines de l'âge classique. De la sorte, ce roman se trouve même à contrarier la prétendue exclusion entre épistémè naturaliste et épistémè mécaniste qu'avait cherché à établir Michel Foucault. Aussi ne doit-on pas s'étonner si, dans un même passage, peuvent être tour à tour sollicitées des références au système de Newton et à Priape, cette figure emblématique de l'exubérance naturaliste: c'est le cas, par exemple, de la description de la première expérience amoureuse de Fanny, alors que les mains de son amant s'étaient égarées sur sa "gorge nue": mais, ajoute-t-elle,

leur rondeur parfaite, leur blancheur, leur fermeté n'étant pas capable de fixer ses mains, il les porta tout à coup sous mes jupes et découvrit le centre d'attraction [...] il tira son Priape et le poussa de toutes ses forces, croyant le lancer dans une voie déjà frayée. Alors je sentis pour la première fois le frottement de cette noble machine. ${ }^{31}$

"Le centre d'attraction", "Priape" et cette "noble machine": en bref, la figure sous laquelle paraissent les sexes suppose la pratique d'une prose à la fois docte et "piquante", "policée" et hardie, que viennent soutenir des métaphores tirées d'au moins deux conceptions distinctes de la nature, qui permettent de solliciter et d'allier tout ensemble le langage de la physique nouvelle et celui de la fable païenne en faveur d'une représentation libertine du corps.

31 Fougeret de Monbron, op. cit., p. 40-41. 